\title{
New aQTL SNPs for the CYP2D6 Identified by a Novel Mediation Analysis of Genome-Wide SNP Arrays, Gene Expression Arrays, and CYP2D6 Activity
}

\author{
Guanglong Jiang, ${ }^{1,2}$ Arindom Chakraborty, ${ }^{1,2}$ Zhiping Wang, ${ }^{1,2}$ Malaz Boustani, ${ }^{3}$ \\ Yunlong Liu, ${ }^{1,2}$ Todd Skaar, ${ }^{4}$ and Lang $\mathrm{Li}^{1,2,4}$ \\ ${ }^{1}$ Department of Medical and Molecular Genetics, Indiana University School of Medicine, Indianapolis, IN 46202, USA \\ ${ }^{2}$ Center for Computational Biology and Bioinformatics, Indiana University School of Medicine, Indianapolis, IN 46202, USA \\ ${ }^{3}$ Regenstrief Institute, Indianapolis, IN 46202, USA \\ ${ }^{4}$ Division of Clinical Pharmacology, Department of Medicine, Indiana University School of Medicine, Indianapolis, \\ IN 46202, USA
}

Correspondence should be addressed to Lang Li; lali@iu.edu

Received 30 August 2013; Accepted 16 September 2013

Academic Editor: Zhongming Zhao

\begin{abstract}
Copyright (C) 2013 Guanglong Jiang et al. This is an open access article distributed under the Creative Commons Attribution License, which permits unrestricted use, distribution, and reproduction in any medium, provided the original work is properly cited.

Background. The genome-wide association studies (GWAS) have been successful during the last few years. A key challenge is that the interpretation of the results is not straightforward, especially for transacting SNPs. Integration of transcriptome data into GWAS may provide clues elucidating the mechanisms by which a genetic variant leads to a disease. Methods. Here, we developed a novel mediation analysis approach to identify new expression quantitative trait loci (eQTL) driving CYP2D6 activity by combining genotype, gene expression, and enzyme activity data. Results. 389,573 and 1,214,416 SNP-transcript-CYP2D6 activity trios are found strongly associated $\left(P<10^{-5}, \mathrm{FDR}=16.6 \%\right.$ and $\left.11.7 \%\right)$ for two different genotype platforms, namely, Affymetrix and Illumina, respectively. The majority of eQTLs are trans-SNPs. A single polymorphism leads to widespread downstream changes in the expression of distant genes by affecting major regulators or transcription factors (TFs), which would be visible as an eQTL hotspot and can lead to large and consistent biological effects. Overlapped eQTL hotspots with the mediators lead to the discovery of 64 TFs. Conclusions. Our mediation analysis is a powerful approach in identifying the trans-QTL-phenotype associations. It improves our understanding of the functional genetic variations for the liver metabolism mechanisms.
\end{abstract}

\section{Introduction}

Genome-wide association studies (GWAS) have identified hundreds of genetic variants associated with complex human diseases, clinical conditions, and traits. These studies have also provided valuable insights into the genetic architecture. Unfortunately, GWAS studies have achieved limited success. The variants discovered usually explain only a small fraction of the overall heritability of the disease [1]. The identification of specific causal genes or mutations from associated regions is a challenge especially for the transacting SNPs which fall either far from genes or a region with many equally plausible causative genes. To make the situation more complicated, sometimes, a single locus can contain multiple independent risk variants (common or rare). Even when a locus is identified by SNP association, the causal mutation itself needs not to be a SNP [2]. For example, GWAS have associated the IRGM gene with Crohn's disease, but a subsequent study showed that the causal mutation is a deletion of the upstream of the promoter affecting tissue-specific expression [3].

There is a substantial gap in understanding the SNP traits associations from a genome-wide association study and the contribution of the locus to a disease. An eQTL approach investigates how the abundance of a gene transcript is directly modified by polymorphism in regulatory elements. The validity of eQTL has been shown in multiple tissue types, 
in which high heritability has been observed in widespread gene transcripts [4-8]. This indicates that genetic influences on gene expression are common. The potential of genomewide eQTL identification has been shown originally in the yeast Saccharomyces cerevisiae [9] and then in humans, animals, and plants $[10,11]$. One of the most important consequences of eQTL mapping is the link that it provides between genetic markers of a disease identified in GWAS and the expression of a specific gene or genes. In particular, the power of these studies depends upon the identification of specific genetic markers that are simultaneously associated with a disease and eQTLs. For example, a study generated genome-wide transcriptional profiles of lymphocyte samples from participants in the San Antonio Family Heart Study and showed that high density lipoprotein cholesterol concentration was influenced by the cis-regulated VNN1 [5, 12]. Another study of postmortem brain tissue identified eQTLs affecting the MAPT and APOE genes, which play an important part in Alzheimer's disease. Utilizing human lymphoblastoid cell lines from the HapMap project, recent pharmacogenomics study reveals novel genetic variants that contribute to etoposide-induced toxicity through affecting gene expression, which included genes that may play a role in cancer (AGPAT2, IL1B, and WNT5B) [13].

The substantial gap between associated regions from GWAS and the identification of causal variations that contribute to a disease might be filled by eQTL analysis. The functional effects of DNA polymorphism on a multifactorial disease can be mediated through several mechanisms. Polymorphisms responsible for the alteration in protein function can have important effects. However, systematic studies of complex diseases with known nonsynonymous SNPs have not yielded many highly significant results, and many associations implicate nonprotein coding regions. It has been shown that $5 \%$ of the human genome is evolutionary conserved and thus functional, whereas less than one-third of this $5 \%$ consists of genes that encode proteins [2]. Variation in gene expression is probably a more important mechanism underlying susceptibility to complex disease $[2,14]$.

Three major different methodologies have been developed and applied to the integrated eQTL and GWAS analyses. The first method focused on the overlapped SNPtrait, SNPgene expression, and gene expression-trait associations [13, 15]. The second method employed the causal inference framework to identify causal model, reactive model, and independent model among SNP, gene expression, and traits. This approach brought in a more molecular mechanism in analyzing the data [16]. The third approach constructed a Bayesian network for the gene expression and traits, while the network construction was weighted by SNP-gene expression correlation [17].

A multistep procedure for identifying key driver of a complex trait has been described by Schadt et al. [16]. Pairwise regressions among genotype variation, gene expression, and complex trait are investigated first. Then the likelihood based causal model selection (LCMS) test is used to identify expression profiles that sit between the complex-trait QTL and complex trait. In this approach, without applying the statistical test for causality, three different models (causal model, reactive model, and independent model) are used. The particular model with the lowest AIC (Akaike information criterion) value is considered to be the best fit for the data. One great advantage of this procedure is that when a correlation between an expression trait and a clinical phenotype does exist, it can distinguish causal, reactive, or independent relationship between them.

1.1. Mediation Analysis. Mediation analysis is the study of the causal chain or the indirect effect, to identify the possible underlying causal mechanisms. Mediation analysis is widely used across many disciplines such as social sciences, to identify the underlying causal mechanisms or to guide the experiments design [18]. A lot of research works focus on the relations between two variables, $X$ and $Y$. Much has been written about two-variable relations, including conditions under which $X$ can be considered a possible cause of $Y$. To this $X \rightarrow Y$ relation, one can add a third variable by using mediation, whereby $X$ causes the mediator, $M$, and $M$ causes $Y$, so $X \rightarrow M \rightarrow Y$ (see Figure 1 ). If $X$ leads to $Y$ through $M$, this is called the indirect effect. Ignoring $M$ leads to incorrect inference about the relation of $X$ and $Y$, since the effect of $M$ is confounded. If $M$ is related to $X$ and/or $Y$, so that information about $M$ improves the prediction of $Y$ by $X$ but does not substantially alter the relation of $X$ to $Y$ when $M$ is included in the analysis, then we consider $M$ as a covariate. In another situation, $M$ may also modify the relation of $X$ to $Y$ such that the relation of $X$ to $Y$ differs at different values of $M$. This is referred to as a moderator or interaction effect (see MacKinnon et al. [18] and references therein).

To establish this indirect relationship, Baron and Kenny [19] proposed a four-step approach in which several regression analyses are conducted, and the significance of the coefficients is examined at each step. In step 1, a simple regression analysis with $X$ predicting $Y$ is conducted (see Figure $1(\mathrm{a})$ ) to test for path $\beta_{1}$ as

$$
Y=\alpha_{1}+\beta_{1} X+\varepsilon_{1}
$$

In step 2, another simple regression analysis is performed with $X$ predicting $M$ to test for path $\beta_{2}$ as

$$
M=\alpha_{2}+\beta_{2} X+\varepsilon_{2} .
$$

And in step 3, the following regression equation is fitted with $M$ predicting $Y$ to test for path $\beta_{3}$ :

$$
Y=\alpha_{3}+\beta_{3} M+\varepsilon_{3} .
$$

Step 2 and step 3 are combined in Figure 1(b). The final step is to conduct a multiple regression analysis with $X$ and $M$ predicting $Y$ as (see Figure 1(c))

$$
Y=\alpha_{4}+\beta_{4} M+\beta_{5} X+\varepsilon_{4} .
$$

In all the above steps, it is assumed that independently, $\varepsilon_{k} \sim N\left(0, \sigma_{k}^{2}\right), k=1,2,3,4$. The purpose of step 1step 3 is to establish that zero-order relationships among the variables exist. One proceeds to step 4 assuming that there are significant relationships from steps 1 through 3 . To 


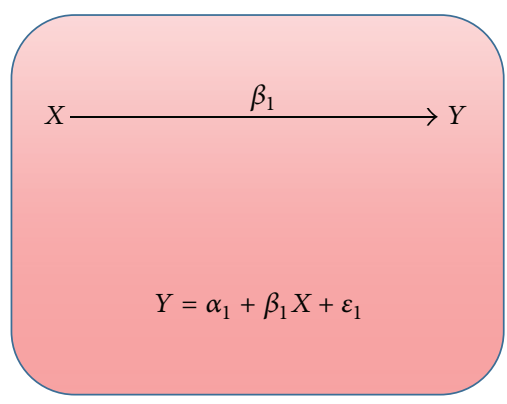

(a)



(b)

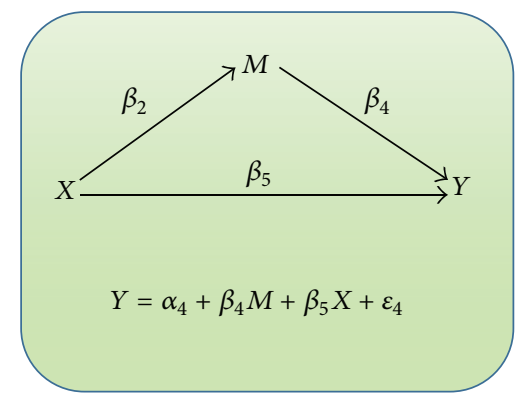

(c)

FIgURE 1: Mediation test.

identify potential causal links between genotype and clinical phenotypes, Huang et al. [20] designed a three-way model based on a stepwise regression analysis with genotype, gene expression, and cytotoxicity data as follows:

\section{S1: SNP is associated with cytotoxicity,}

S2: SNP is associated with gene expression,

S3: gene expression is associated with cytotoxicity.

Theoretical evidence in the form of "Causality Equivalence Theorem" has been proposed by Chen et al. [21] to establish causal relationship. According to the theorem, under the assumption that $X$ is randomized, the following conditions are needed to establish a causal relation:

\section{C1: $X$ and $M$ are associated, \\ C2: $X$ and $Y$ are associated, \\ C3: $X$ is independent of $Y \mid M$.}

If both $X$ and $M$ are significant predictors of $Y$, then partial mediation is achieved, whereas if $X$ is no longer significant when $M$ is controlled, this supports the condition of full mediation. However, there are some limitations of this test as mentioned by MacKinnon et al. [22]. This includes a low power to detect mediation and biased estimates. It does not test for the significance for the indirect pathway. An alternative and preferable approach to estimate the indirect effect is by multiplying two regression coefficients, $\beta_{2} \times \beta_{4}$ [23].

In this paper, we introduce a new method, mediation analysis, which is somewhere between the overlap analysis (the first method) and causal inference (the second method). We use the human liver consortium data to demonstrate its application and performance. We use genome-wide genotype and gene expression data to explore functional mutation for an important pharmacogene, CYP2D6, which is a member of the cytochrome P450 mixed-function oxidase system and is responsible for the metabolism of $25 \%$ of all drugs on the market.

\section{Material and Methods}

2.1. Human Liver Cohort Dataset. Human liver cohort (HLC) data are collected from Sage Bionetworks Repository and
Gene Expression Omnibus (GEO) database as described in the literature [17]. The dataset includes 2 genotype arrays (Illumina Sentrix human Hap650Y genotyping beadchip and Affymetrix 500 K genotyping array), gene expressions (30,128 probes $\times 466$ samples) and enzyme activities (10 activity measurements of 9 enzymes $\times 488$ samples), and demographic information. Genotype data for 219 Illumina and 214 Affymetrix that are publicly accessible are used. Patients with genotyping call rate less than 95\% are removed from further analysis. This filtration reduces the sample sizes to 204 and 207 for Affymetrix and Illumina platforms, respectively. 167 Illumina genotyping has both gene expression data and enzyme activity data. In case of Affymetrix platform, 180 samples overlapped with gene expression and enzyme activity data.

SNPs whose genotyping call rate are less than $95 \%$ or Hardy-Weinberg equilibrium tests are significant $(P<0.001)$ or minor allele frequency $<10 \%$ are discarded. For Affymetrix platform, 214,399 SNPs, and for Illumina, 471,394 SNPs are used for mediation and eQTL analysis. Enzyme activity and gene expression data are corrected with age and gender and then are normalized with normal quartile normalization.

2.2. Mediation Analysis. The mediation analysis method is developed to assess the indirect effects of genetic variant to CYP2D6 activity mediated by gene expressions. The tests are performed by parallel programming using $\mathrm{C}$ and $\mathrm{MPICH}$. The computations are run on a Linux cluster computing environment with 200 compute nodes, and each node takes around 36 hours.

MacKinnon proposed a permutation test for mediation that makes use of the permutation-of-raw-data approach for testing a regression coefficient $[22,24]$. It is referred to as the permutation test of $\beta_{2} \times \beta_{4}$. To test for regression coefficients, permutation tests have been applied in several ways [24-26]. Applying this method requires, first, that the regression models in (2) and (4) are estimated for the original, nonpermuted data to find the values of $\beta_{2}$ and $\beta_{4}$. Values of the outcome variable, $Y$, are then permuted $10^{9}$ times and reassigned to nonpermuted scores on the predictor, $X$, and mediator, $M$, to create many permuted samples. The permuted $Y$ values, labeled $Y^{+}$, are then regressed on the nonpermuted $X$ and $M$ values in each permuted sample 
TABLE 1: Mediation analysis.

\begin{tabular}{lllccrc}
\hline $\begin{array}{l}\text { Genotype } \\
\text { dataset }\end{array}$ & Enzyme & SNP effect & $\begin{array}{c}\text { No. of sig. } \\
\text { trios } \\
P<10^{-5}\end{array}$ & $\begin{array}{c}\text { No. of sig. } \\
\text { SNPs } \\
P<10^{-5}\end{array}$ & $\begin{array}{c}\text { No. of sig. } \\
\text { exp }\end{array}$ & $\begin{array}{c}\text { FDR } \\
\text { (trios) }\end{array}$ \\
\hline Affymetrix & CYP2D6 & Gene dose & 389,573 & 103,369 & 3,545 & $16.63 \%$ \\
Illumina array & CYP2D6 & Gene dose & $1,214,416$ & 251,738 & 4,770 & $11.73 \%$ \\
\hline
\end{tabular}

TABLE 2: eQTL analysis.

\begin{tabular}{lccc}
\hline $\begin{array}{l}\text { Genotype } \\
\text { dataset }\end{array}$ & $\begin{array}{c}\text { No. of pairs } \\
P<10^{-5}\end{array}$ & $\begin{array}{c}\text { No. of SNPs } \\
\text { Correlated with }>1 \text { gene (total SNPs) }\end{array}$ & $\begin{array}{c}\text { No. of SNPs } \\
\text { Correlated with }>20 \text { genes }\end{array}$ \\
\hline Affymetrix & 65,763 & $28,089(214,399)$ & 295 \\
Illumina & 154,546 & $63,643(471,394)$ & 724 \\
\hline
\end{tabular}

(as in (4)), and the coefficient for $M$ in each permuted sample is labelled $\beta_{4}^{*}$. Similarly, values of the mediator, $M$, are permuted $10^{9}$ times and reassigned to values of the predictor $X$ to create many permuted samples. The permuted $M$ values, labeled $M^{+}$, are regressed on $X$ in each permuted sample (as in (2)), and the coefficient for $X$ in each permuted sample is labelled $\beta_{2}^{+}$. Finally, corresponding pairs of $\beta_{2}^{+}$and $\beta_{4}^{+}$values are multiplied to yield $\beta_{2}^{+} \beta_{4}^{+}$, and $\widehat{\beta}_{2} \widehat{\beta}_{4}$, the estimate of the mediated effect from the original data, is compared to the distribution of $\beta_{2}^{+} \beta_{4}^{+}$to perform a test of the null hypothesis of no mediation.

The mediated effect is estimated by the product of coefficients $\left(\beta_{2} \times \beta_{4}\right)$ then divided by its standard error, which is derived by Sobel [23], under the assumption of multivariate normality for the standard error of the indirect effect, using the multivariate delta method as

$$
\sigma_{\beta_{2} \beta_{4}}=\sqrt{\beta_{2}^{2} \sigma_{4}^{2}+\beta_{4}^{2} \sigma_{2}^{2}} .
$$

Hence, the test statistics are

$$
\Delta_{\text {Indirrect effect }}=\frac{\widehat{\beta}_{2} \widehat{\beta}_{4}}{\operatorname{se}\left(\widehat{\beta}_{2} \widehat{\beta}_{4}\right)} .
$$

2.3. Genome-Wide Association Based on Mediation Analysis. The huge sizes of SNP and gene expression probes in mediation analysis introduce problems related to multiple hypotheses testing. False discovery rate (FDR) is used to control type I error for multiple testing. FDR is calculated as

$$
\mathrm{FDR}=\frac{\# \text { significance by chance }}{\# \text { significance results }} \text {. }
$$

A stringent threshold is needed to avoid high FDR. Comparing to cis-acting variations, more transacting variations are detected by GWAS. In GWAS analysis, transeffects are usually weaker than cis-effects but are more numerous than the latter [14]. The trans-acting SNPs having smaller effects than cis-acting SNPs are more likely to be missed if more stringent threshold is applied.

2.4. eQTL Analysis. Transcript abundance is highly heritable in human populations and can be considered as a quantitative trait and be mapped to particular genomic loci, known as expression quantitative loci (eQTL). Not only gene expression is itself a complex trait, but also it acts as an intermediate phenotype between genetic loci and higher level cellular or clinical phenotypes, such as disease risk or individual drug response [27].

Linear model is fitted with genome-wide genotype and gene expression profiles. eQTL analysis is run in parallel on the same computing cluster with $\mathrm{R}$ language program. eQTL hotspots are defined as SNPs enriched in correlations with expression profiles across the genome (SNPs correlated with at least 20 gene expression profiles). The correlation $P$ values between SNP and expression probe less than $10^{-5}$ are considered to be significant and used for hotspot analysis. To test the enrichment of significant correlation between eQTL and all gene expression probes, exact binomial tests are conducted and corrected with Bonferroni method, and the corrected $P$ values are used as the enrichment scores.

\section{Results}

3.1. Mediation Analysis. The result of mediation analysis is summarized in Table 1 . To find the significant trios, $P$ values less than $10^{-5}$ are considered. Using the same criteria for both platforms, the number of significant trios differs. For Affymetrix platform, we have 389,573 trios having $P$ values less than $10^{-5}$. For the other platform, this number is $1,214,416$. The FDR for Illumina platform is found to be $11.73 \%$, whereas for Affymetrix platform it is a bit higher $(16.63 \%)$.

3.2. eQTL Analysis. In Table 2, the result corresponding to eQTL analysis of the HLC data is reported. The Affymetrix dataset has 214,399 SNPs after the implementation of the quality control out of which 28,089 are correlated with at least one gene at $P<10^{-5}$ significance level, and there are total 65,763 SNP-gene pairs significantly correlated. 295 SNPs are correlated with at least 20 genes. Those 295 hotspots are used to check for overlapping with the results of mediation analysis. 289 eQTL hotspots are found correlated with 1542 gene expression profiles at $P<10^{-5}$ significance level (Table 3). In contrast, Illumina dataset has higher quality 
TABLE 3: QTL overlapping.

\begin{tabular}{|c|c|c|c|c|}
\hline \multirow{3}{*}{ Overlapping } & \multicolumn{2}{|c|}{ Affymetrix } & \multicolumn{2}{|c|}{ Illumina } \\
\hline & $\begin{array}{l}\text { No. of eQTL } \\
\text { hotspots }\end{array}$ & $\begin{array}{l}\text { No. of mediation } \\
\text { trios }\end{array}$ & $\begin{array}{l}\text { No. of eQTL } \\
\text { hotspots }\end{array}$ & $\begin{array}{l}\text { No. of mediation } \\
\text { trios }\end{array}$ \\
\hline & 295 & 389,573 & 724 & $1,214,416$ \\
\hline No. of eQTL hotspot trios (No. of SNPs, No. of genes) & \multicolumn{2}{|c|}{$9,296(289,1,542)$} & \multicolumn{2}{|c|}{$34,880(719,2,444)$} \\
\hline
\end{tabular}

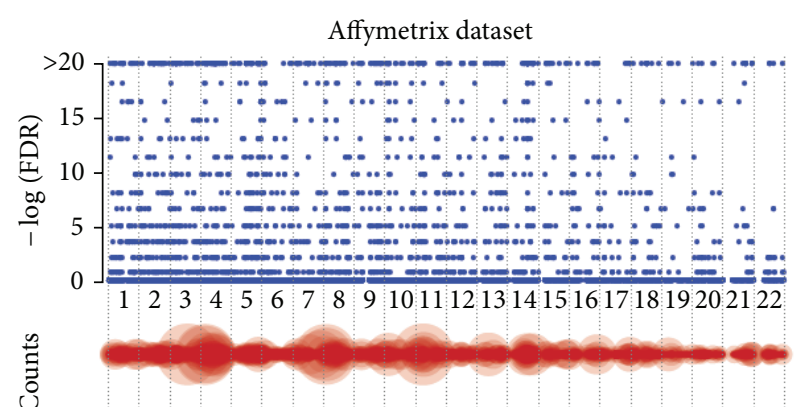

12234556788910111213141516171819202122

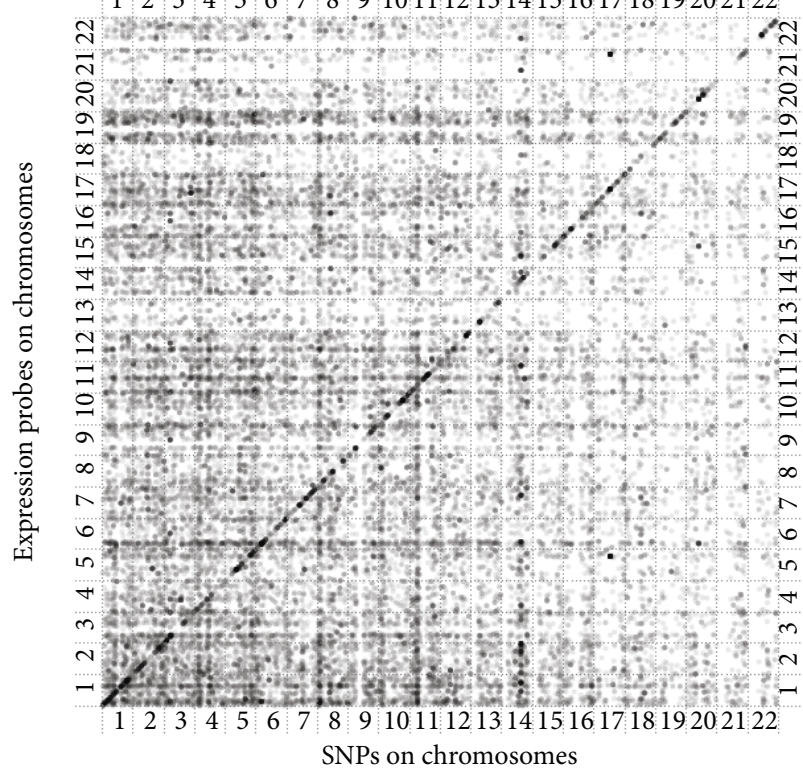

(a)

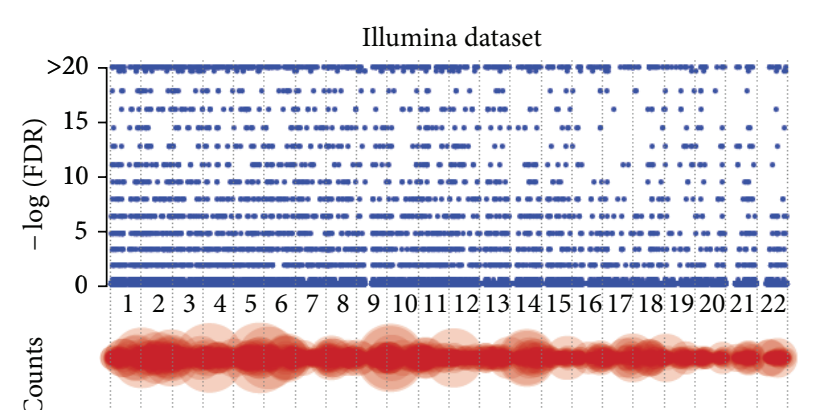

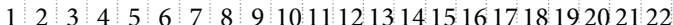
त

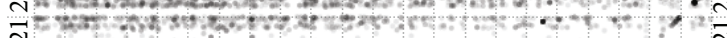
i

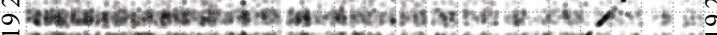

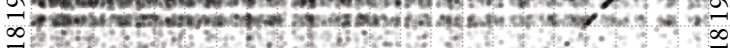
-

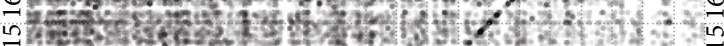
in m. crane 9.5. o 5 की a thats of $\infty 3^{2}$.

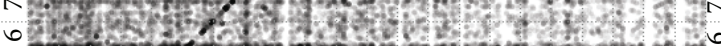
in

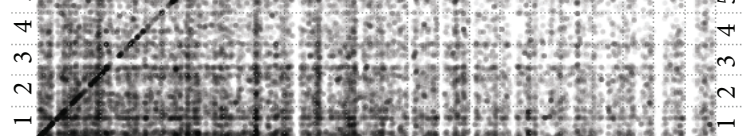

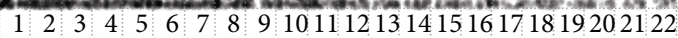
SNPs on chromosomes

(b)

FIgURE 2: eQTL visualization. The main plot at the bottom is the scatter plot of the eQTL-transcript association. Each dot denotes a significant association between a SNP and a transcript $\left(P\right.$ value $\left.<10^{-5}\right)$. Gray color shows the level of significance where dark means more significant association. SNPs are arranged according to their chromosomal loci along the $X$-axis from chromosome 1 to 22 , and genes are arranged along $Y$-axis in the same way. The dots along diagonal line indicate cis-eQTLs, otherwise, trans-eQTLs. The counts plot in the middle gives the number of genes that a SNP correlated with significantly $\left(P\right.$ value $\left.<10^{-5}\right)$. Large size means more genes associated with that SNP. The $-\log _{10}$ (FDR) plot at the top presents the enrichment score of a SNP associated with multiple transcripts comparing with that by chance. SNP has a large circle in counts plot and a high enrichment score in - $\log _{10}$ (FDR) plot which indicates eQTL hotspots.

with more SNPs passed quality control tests. Out of 471,394 SNPs, 63,643 SNPs are found to be correlated with at least one gene at $P<10^{-5}$ significance level. Numbers of SNPs that are correlated with at least 20 genes are found to be 724 , and 719 of the hotspots are significantly correlated with 2,444 genes in mediation analysis (Table 3). In Figure 2, a pictorial depiction of this eQTL analysis is given for both platforms. The significant SNP-expression pairs $\left(P<10^{-5}\right)$ are plotted as a dot according to the locations of the SNP and the gene on 22 chromosomes along $X$-axis and $Y$-axis. The grey colors show the level of significance, with darker dots representing smaller $P$ values. The counts of significant SNP-expression pairs and $-\log _{10}$ (FDR) for a given SNP are also plotted above the eQTL image. For each SNP, the count gives the number of genes that are correlated with this particular SNP, as the larger radius of the circle indicates that the SNP is correlated with more genes. In that case, it may be considered to be a potential eQTL hotspot. The dots along diagonal line indicate 
TABLE 4: Functional annotations of the mediators.

\begin{tabular}{lcc}
\hline Types & $\begin{array}{c}\text { Affymetrix no. } \\
\text { Mediator genes }\end{array}$ & $\begin{array}{c}\text { Illumina no. } \\
\text { Mediator genes }\end{array}$ \\
\hline Cytokine & 5 & 7 \\
Enzyme & 246 & 368 \\
G-protein coupled & 17 & 20 \\
receptor & 5 & 11 \\
Growth factor & 13 & 18 \\
Ion channel & 52 & 62 \\
Kinase & 5 & 7 \\
Ligand-dependent nuclear & 373 & 596 \\
receptor & 31 & 39 \\
Other & 15 & 21 \\
Peptidase & 82 & 118 \\
Phosphatase & 6 & 10 \\
Transcription regulator & 12 & 16 \\
Translation regulator & 77 & 127 \\
Transmembrane receptor & 939 & 1420 \\
Transporter & &
\end{tabular}

cis-effects. It can be seen that cis-eQTLs have bigger effect on expression profile compared to trans-eQTLs.

3.3. Functional Analysis of Hotspots Mediators. 1,542 and 2,444 hotspot mediators from Affymetrix and Illumina platforms annotated to 1,388 and 2,187 unique genes separately. 939 and 1420 genes are successfully mapped in Ingenuity database for two platforms. The functional annotations of these genes are summarized in Table 4. Five (CCL16, CCL20, CMTM5, IL6, and SPP1) and 7 (CCL16, CCL20, CKLF, CKLFSF5, EPO, FAM3C, and SPP1) cytokines, 5 (AR, NR1I2, NR1I3, NR2F6, and PPARA) and 7 (AR, ESR1, NR1I2, NR1I3, PPARA, RORA, and RORC) ligand-dependent nuclear receptors, and 80 and 113 transcription regulators are found to mediate the relationship between genetic variant and CYP2D6 activity for Affymetrix and Illumina platforms. 64 transcription regulators overlapped between the two platforms (Gene List 1). Among the 64 transcription factors predicted mediateding genetic regulation of CYP2D6 activity, YY1 is reported putatively binding to gene CYP2D6 promoter region and regulating the expression of CYP2D6 and CYP2D4 $[28,29]$.

\section{Conclusion}

Cytochrome P450 constitutes a large subfamily of enzymes that plan an important role in the metabolism of endogenous compounds and the activation of chemical carcinogens. In this work, the regulations of P450 expression and activities have been intensely studied. Several other studies have found that P450 are subject to regulation by liverenriched transcription factors, cytokines, and nuclear receptors. Our study provides some new clues on the regulation of CYP2D6 enzyme activity. Our mediation analysis is a powerful approach in identifying the trans-SNP-phenotype associations. We found a rich class of functional categories of mediators that potentially control the CYP2D6 activities, which include many new transcription factors. This method has some limitations too. In this work, the relationship between genetic variants, gene expression, and phenotype is assumed to be a simple one. However, in most of the situations, this relationship may become very complex. More sophisticated methods are required to analyze those complex models. In mediation analysis, we are only interested in testing the product of two regression coefficients. Mediation analysis cannot provide causal inference. The mediation analysis assumes that there is some causal relationship. It will be necessary to test for the assumption. We need to be extra cautious about drawing the conclusion of the causal relationship. Our studies provide insights into the comprehension of the complex regulatory network of CYP2D6 and improve our understanding of the functional genetic variations for the liver metabolism mechanisms.

\section{Genes List}

64 TFs overlapped between Affymetrix and Illumina datasets, including AATF, ALYREF, ARHGAP35, ASB8, ATF4, CBX4, CEBPG, CSDA, DDIT3, E2F5, ETV7, FOXN3, FOXN3, FUBP1, GPS2, HDAC10, HMGN1, ID1, INVS, IRF9, KANK1, KAT2B, KHDRBS1, KLF12, MAF, MAML2, MEIS2, MLXIPL, MXD4, MYBBP1A, MYCL1, NCOA7, NCOR1, NFIA, NFKB2, NFYA, NOLC1, NPM1, PEX14, PYCARD, SAP18, SATB1, SIM2, SLC2A4RG, SMARCC1, SNAI3, SNW1, SOX5, TCERG1, TCF7L2, TEAD3, TEAD4, TFDP2, TFEB, TOB1, TP53, YWHAB, YY1, ZGPAT, ZHX3, ZKSCAN1, ZNF132, ZNF256, and ZNF263.

\section{Abbreviations \\ GWAS: Genome-wide association study \\ eQTL: Expression quantitative trait loci \\ aQTL: Enzyme activity quantitative trait loci \\ FDR: False discovery rate \\ HLC: Human liver cohort \\ TFs: Transcription factors.}

\section{Conflict of Interests}

The authors have declared that no competing interests exist.

\section{Acknowledgments}

This work is supported by the US National Institutes of Health Grant R01 GM74217 (Lang Li), R01 GM088076 (Todd Skaar), and AHRQ Grant R01HS019818-01 (Malaz Boustani). The content is solely the responsibility of the authors and does not necessarily represent the official views of the Agency for Healthcare Research and Quality. 


\section{References}

[1] J. H. Moore, F. W. Asselbergs, and S. M. Williams, "Bioinformatics challenges for genome-wide association studies," Bioinformatics, vol. 26, no. 4, Article ID btp713, pp. 445-455, 2010.

[2] D. Altshuler, M. J. Daly, and E. S. Lander, "Genetic mapping in human disease," Science, vol. 322, no. 5903, pp. 881-888, 2008.

[3] S. A. McCarroll, A. Huett, P. Kuballa et al., "Deletion polymorphism upstream of IRGM associated with altered IRGM expression and Crohn's disease," Nature Genetics, vol. 40, no. 9, pp. 1107-1112, 2008.

[4] A. L. Dixon, L. Liang, M. F. Moffatt et al., "A genome-wide association study of global gene expression," Nature Genetics, vol. 39, no. 10, pp. 1202-1207, 2007.

[5] H. H. H. Göring, J. E. Curran, M. P. Johnson et al., "Discovery of expression QTLs using large-scale transcriptional profiling in human lymphocytes," Nature Genetics, vol. 39, no. 10, pp. 12081216, 2007.

[6] S. A. Monks, A. Leonardson, H. Zhu et al., "Genetic inheritance of gene expression in human cell lines," American Journal of Human Genetics, vol. 75, no. 6, pp. 1094-1105, 2004.

[7] E. Petretto, J. Mangion, N. J. Dickens et al., "Heritability and tissue specificity of expression quantitative trait loci," PLoS Genetics, vol. 2, no. 10, Article ID e172, 2006.

[8] P. M. Visscher, W. G. Hill, and N. R. Wray, "Heritability in the genomics era-concepts and misconceptions," Nature Reviews Genetics, vol. 9, no. 4, pp. 255-266, 2008.

[9] R. B. Brem, G. Yvert, R. Clinton, and L. Kruglyak, "Genetic dissection of transcriptional regulation in budding yeast," Science, vol. 296, no. 5568, pp. 752-755, 2002.

[10] M. V. Rockman and L. Kruglyak, "Genetics of global gene expression," Nature Reviews Genetics, vol. 7, no. 11, pp. 862-872, 2006.

[11] E. E. Schadt, S. A. Monks, T. A. Drake et al., "Genetics of gene expression surveyed in maize, mouse and man," Nature, vol. 422, no. 6929, pp. 297-302, 2003.

[12] A. J. Myers, J. R. Gibbs, J. A. Webster et al., "A survey of genetic human cortical gene expression," Nature Genetics, vol. 39, no. 12, pp. 1494-1499, 2007.

[13] R. S. Huang, S. Duan, W. K. Bleibel et al., "A genomewide approach to identify genetic variants that contribute to etoposide-induced cytotoxicity," Proceedings of the National Academy of Sciences of the United States of America, vol. 104, no. 23, pp. 9758-9763, 2007.

[14] W. Cookson, L. Liang, G. Abecasis, M. Moffatt, and M. Lathrop, "Mapping complex disease traits with global gene expression," Nature Reviews Genetics, vol. 10, no. 3, pp. 184-194, 2009.

[15] N. Niu, Y. Qin, B. L. Fridley et al., "Radiation pharmacogenomics: a genome-wide association approach to identify radiation response biomarkers using human lymphoblastoid cell lines," Genome Research, vol. 20, no. 11, pp. 1482-1492, 2010.

[16] E. E. Schadt, J. Lamb, X. Yang et al., "An integrative genomics approach to infer causal associations between gene expression and disease," Nature Genetics, vol. 37, no. 7, pp. 710-717, 2005.

[17] X. Yang, B. Zhang, C. Molony et al., "Systematic genetic and genomic analysis of cytochrome P450 enzyme activities in human liver," Genome Research, vol. 20, no. 8, pp. 1020-1036, 2010.

[18] D. P. MacKinnon, C. M. Lockwood, C. H. Brown, W. Wang, and J. M. Hoffman, "The intermediate endpoint effect in logistic and probit regression,” Clinical Trials, vol. 4, no. 5, pp. 499-513, 2007.
[19] R. M. Baron and D. A. Kenny, "The moderator-mediator variable distinction in social psychological research. Conceptual, strategic, and statistical considerations," Journal of Personality and Social Psychology, vol. 51, no. 6, pp. 1173-1182, 1986.

[20] B. Huang, S. Sivaganesan, P. Succop, and E. Goodman, "Statistical assessment of mediational effects for logistic mediational models," Statistics in Medicine, vol. 23, no. 17, pp. 2713-2728, 2004.

[21] L. S. Chen, F. Emmert-Streib, and J. D. Storey, "Harnessing naturally randomized transcription to infer regulatory relationships among genes," Genome Biology, vol. 8, no. 10, Article ID R219, 2007.

[22] D. P. MacKinnon, A. J. Fairchild, and M. S. Fritz, "Mediation analysis," Annual Review of Psychology, vol. 58, pp. 593-614, 2007.

[23] M. E. Sobel, "Asymptotic confidence intervals for indirect effects in structural equation models," Sociological Methodology, vol.13, pp. 290-312, 1982.

[24] B. F. J. Manly, Randomization, Bootstrap and Monte Carlo Methods in Biology, vol. 70, Chapman \& Hall, New York, NY, USA, 1997.

[25] M. J. Anderson and P. Legendre, "An empirical comparison of permutation methods for tests of partial regression coefficients in a linear model," Journal of Statistical Computation and Simulation, vol. 62, no. 3, pp. 271-303, 1999.

[26] C. J. F. Terbraak, "Permutation versus bootstrap significance tests in multiple-regression and Anova," in Bootstrapping and Related Techniques, vol. 376 of Lecture Notes in Economics and Mathematical Systems, pp. 79-85, 1992.

[27] E. R. Gamazon, W. Zhang, A. Konkashbaev et al., "SCAN: SNP and copy number annotation," Bioinformatics, vol. 26, no. 2, pp. 259-262, 2010.

[28] X. L. Gong, Y. Liu, X. Zhang et al., "Systematic functional study of cytochrome P450 2D6 promoter polymorphisms in the Chinese Han population," Plos One, vol. 8, no. 2, Article ID e57764, 2013.

[29] D. Mizuno, Y. Takahashi, T. Hiroi, S. Imaoka, T. Kamataki, and Y. Funae, "A novel transcriptional element which regulates expression of the CYP2D4 gene by Oct-1 and YY-1 binding," Biochimica et Biophysica Acta, vol. 1627, no. 2-3, pp. 121-128, 2003. 

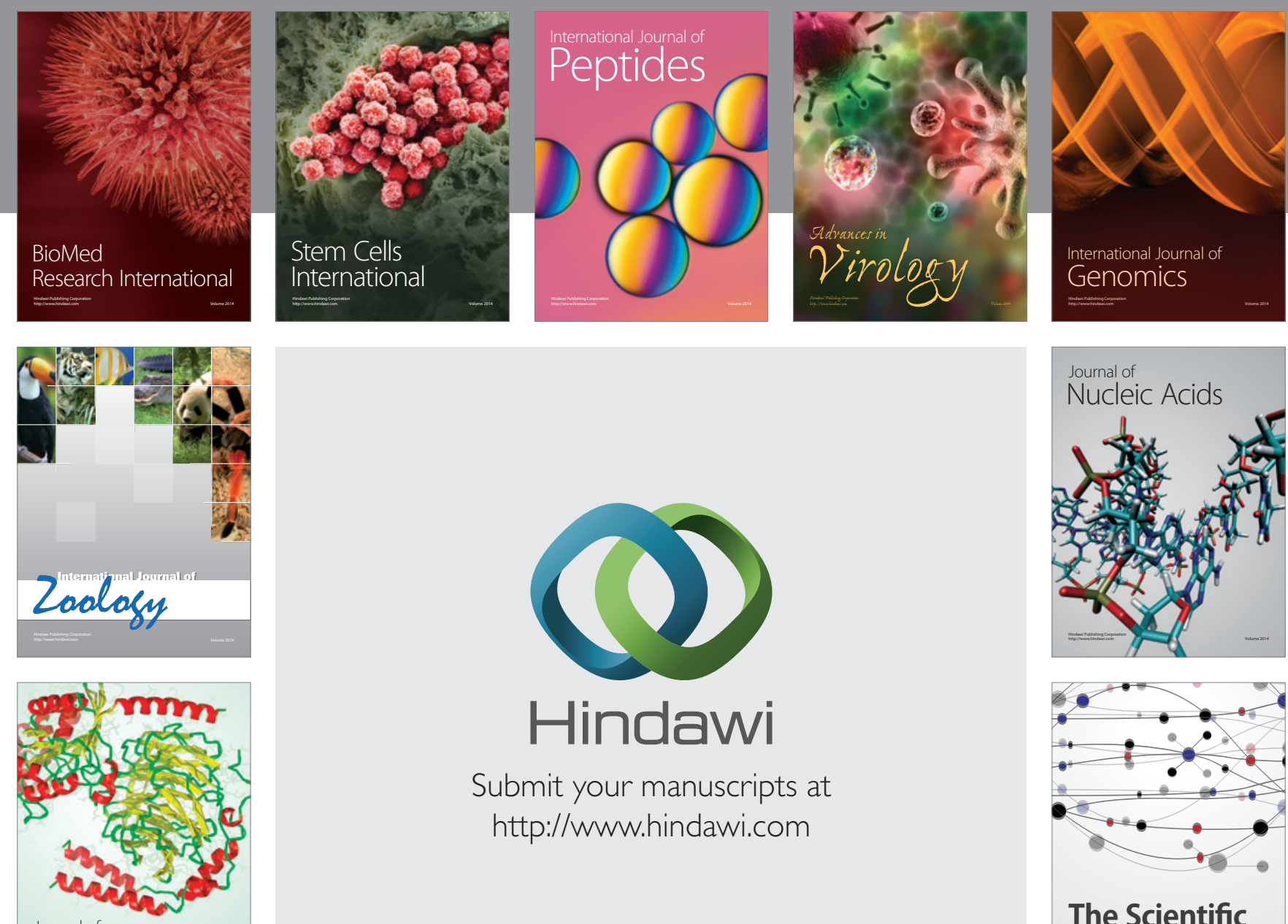

Submit your manuscripts at

http://www.hindawi.com

Journal of
Signal Transduction
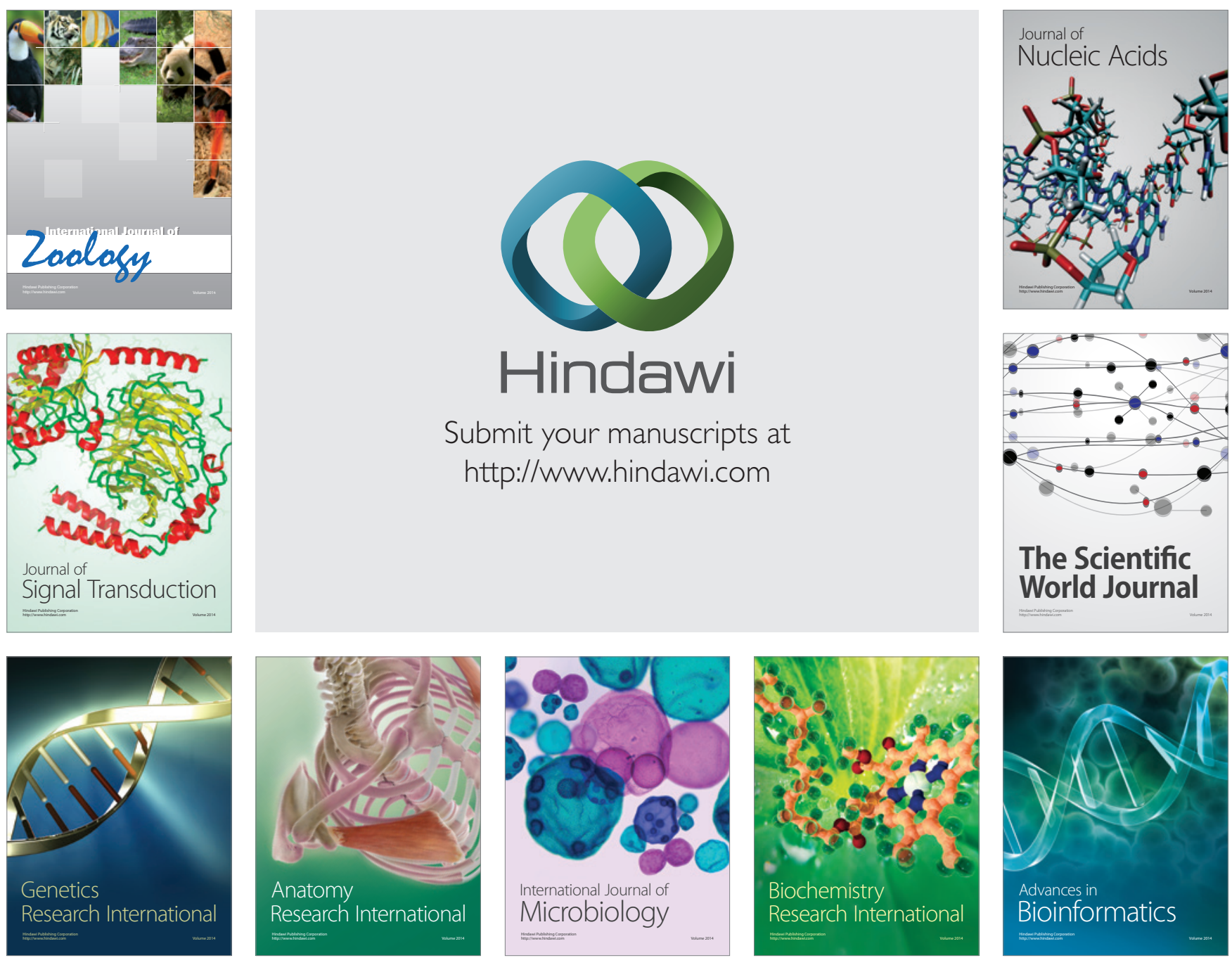

The Scientific World Journal
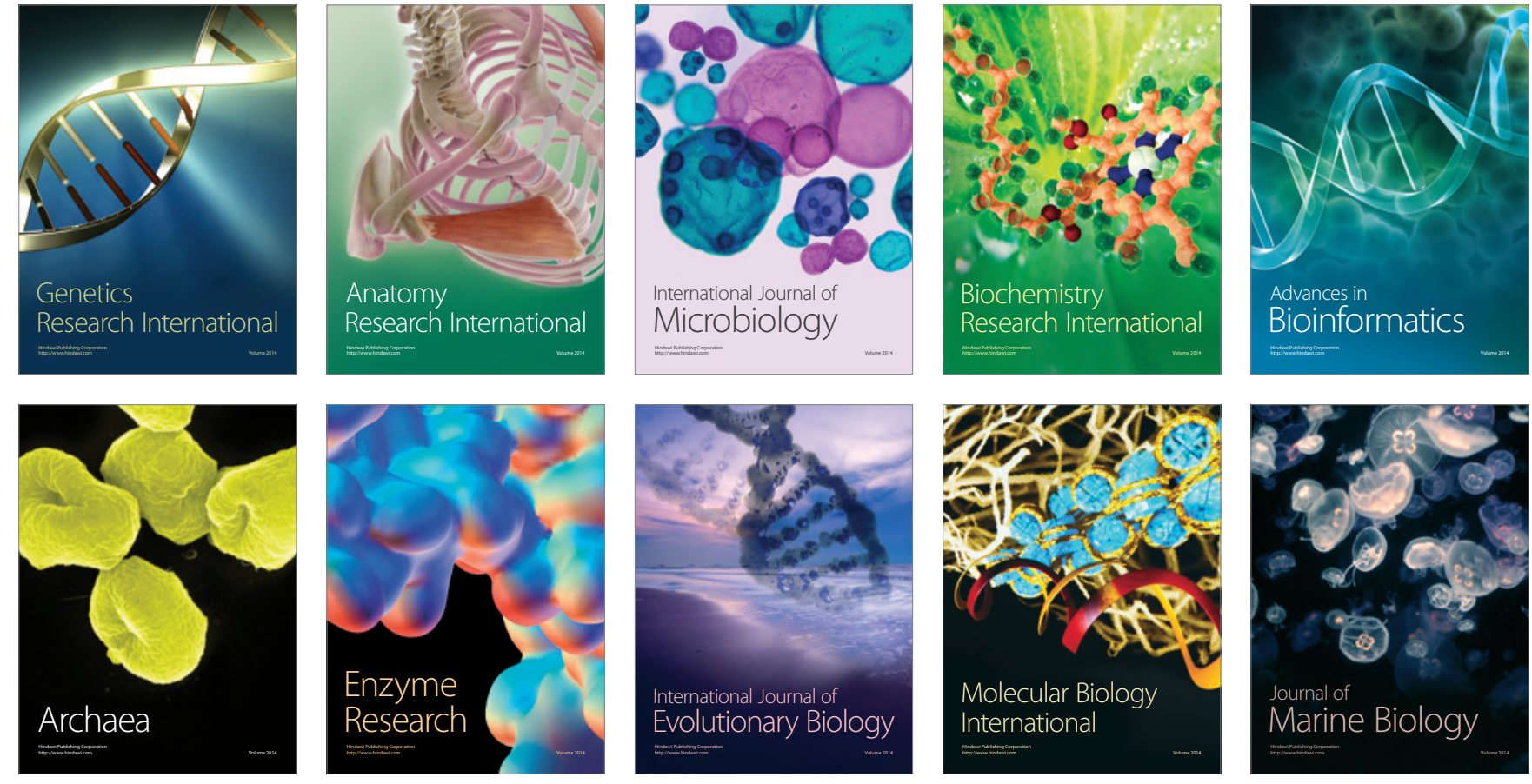